\title{
Visual hemispatial inattention: stimulus parameters and exploratory strategies
}

\author{
SANDRA WEINTRAUB, M MARSEL MESULAM \\ From the Division of Neuroscience and Behavioural Neurology and Charles A. Dana Research Institute, Beth \\ Israel Hospital and Harvard Medical School, Boston, Massachusetts, USA
}

SUMMARY Patients with unilateral hemispheric lesions were given visual target cancellation tasks. As expected, marked contralateral and less severe ipsilateral visual inattention were observed in patients with right-sided cerebral lesions whereas those with left-sided lesions showed only mild contralateral neglect. Stimulus material (shapes vs letters) and array (random vs structured) interacted in a complex manner to influence target detection only in patients with right-sided lesions. Furthermore, the search strategy of these patients tended to be erratic, particularly when the stimuli were in an unstructured array. A structured array prompted a more systematic and efficient search. It appears, therefore, that stimulus content and spatial array affect neglect behaviour in patients with right-sided lesions and that a lack of systematic visual exploration within the extrapersonal space is one factor that contributes to visual hemispatial inattention.

The severity of visual hemispatial neglect manifested by patients with unilateral cerebral lesions can vary depending on the nature of the stimuli being examined. ${ }^{1-3}$ Many studies investigating stimulus parameters and neglect have concentrated on differences related to stimulus content, such as, verbal vs nonverbal symbols. Leicester et al $^{1}$ reported that neglect was more prominent in patients with right hemispheric lesions on match-to-sample tasks in which form discrimination was a factor while in patients with left hemispheric lesions tasks requiring letter discrimination were more likely to elicit neglect. Using target cancellation tasks, Heilman and Watson ${ }^{3}$ reported that patients with right cerebral lesions showed more hemispatial neglect for words than for slanted lines. This finding, directly opposed to that of Leicester et al, was cited in support of Kinsbourne's ${ }^{45}$ hypothesis that stimuli preferentially activating one cerebral hemisphere (that is, verbal-left vs spatial-right) would enhance orientation to the contralateral hemispace, reducing neglect in that field. More recently, in a large scale study with right and left hemisphere damaged patients, Caplan ${ }^{6}$ failed to replicate Heilman and Watson's results and reported instead that patients with right cerebral

Address for reprint requests: S. Weintraub, $\mathrm{PhD}$, Behavioural Neurology Unit, K-225, Beth Israel Hospital, 330 Brookline Avenue, Boston, MA02215, USA

Received 10 July 1987 and in revised form 10 June 1988.

Accepted 20 June 1988 lesions showed a similar degree of neglect with both verbal and nonverbal stimuli.

In the present study we re-examined the effect of stimulus material on the severity of neglect by recording the number of target omissions and the time spent to detect single targets on visual cancellation tasks differing in stimulus material (letters vs shapes). Since visual hemispatial neglect may in part also be related to defective search strategies,' we introduced the variable of stimulus array (random vs structured), a factor that has not been previously examined.

\section{Methods}

Subjects Subjects consisted of eight patients with unilateral right hemispheric lesions (mean age 65.13 yr, SEM 4.46), eight with unilateral left hemispheric lesions (mean age 58.75 yr, SEM 3.28) and nine normal control subjects (mean age $60.33 \mathrm{yr}$, SEM 2.80). The three groups did not differ with respect to age $[F(2,22)=0.883, p>0.05]$. All subjects were right-handed and, with the exception of one patient (Subject No 10, table 1), had suffered cerebrovascular accidents. The patients were not preselected and were drawn consecutively from the inpatient Neurological Service and the outpatient Behavioural Neurology Unit at the Beth Israel Hospital. Aphasic patients were not excluded since all could understand the simple instructions and identify single letters. There was no significant difference between the patient groups with respect to time elapsed between the onset of illness and testing [mean right lesions 10.59 weeks, SEM 5.40; mean left lesions 23.09 weeks, SEM 19.06; $t(14)=0.630$, p > 0.05, twotailed]. With the exception of subject No 1 , all subjects were 

tasks

\begin{tabular}{|c|c|c|c|c|c|c|c|}
\hline Subject No & Age & Sex & Duration * & Hemiparesis & Hemisensory & Hemianopia & Visual extinction \\
\hline \multicolumn{8}{|l|}{ Left lesion } \\
\hline 1 & 53 & $\mathbf{M}$ & 156.00 & + & - & - & - \\
\hline 2 & 54 & $\mathbf{M}$ & 16.00 & + & - & - & - \\
\hline 3 & 59 & $\mathbf{F}$ & 0.29 & - & - & + & $\mathbf{N} / \mathbf{A}$ \\
\hline 4 & 46 & $\mathbf{F}$ & $4 \cdot 00$ & + & + & - & + \\
\hline 5 & 70 & $\mathbf{F}$ & 6.00 & + & - & - & - \\
\hline 6 & 54 & $\mathbf{F}$ & 1.00 & + & + & - & - \\
\hline 7 & 60 & $\mathbf{M}$ & 0.43 & + & + & + & $\mathbf{N} / \mathbf{A}$ \\
\hline 8 & 74 & $\mathbf{F}$ & $1 \cdot 00$ & + & + & + & $\mathbf{N} / \mathbf{A}$ \\
\hline \multicolumn{8}{|l|}{ Right lesion } \\
\hline 9 & 75 & $\mathbf{F}$ & 0.43 & + & - & - & + \\
\hline 10 & 68 & $\mathbf{M}$ & $40 \cdot 00$ & - & - & - & - \\
\hline 11 & 42 & $\mathbf{F}$ & 0.57 & - & - & + & $\mathbf{N} / \mathbf{A}$ \\
\hline 12 & 76 & $\mathbf{F}$ & $12 \cdot 00$ & + & - & - & + \\
\hline 13 & 66 & $\mathrm{~F}$ & 28.00 & + & + & - & - \\
\hline 14 & 80 & $\mathbf{M}$ & $2 \cdot 00$ & + & - & - & + \\
\hline $\begin{array}{l}15 \\
16\end{array}$ & $\begin{array}{l}58 \\
56\end{array}$ & $\underset{\mathbf{F}}{\mathbf{M}}$ & 0.71 & $\begin{array}{l}+ \\
t\end{array}$ & $\begin{array}{l}+ \\
+\end{array}$ & $\bar{t}$ & $\stackrel{+}{\mathbf{N}}$ \\
\hline 16 & & $F$ & 1.00 & + & + & + & $\mathbf{N} / \mathbf{A}$ \\
\hline
\end{tabular}

*Duration is calculated in weeks elapsed between onset of illness and testing.

$\mathbf{N} / \mathbf{A}=$ Not applicable.

examined within 10 months of the onset of illness. The normal control group consisted of patients' spouses and other volunteers with no significant medical, neurological or psychiatric history.

All patients underwent a thorough neurological examination including CT scan which revealed that lesion size was comparable in the two groups. The frequency of hemiparesis, hemianopia and hemisensory loss in each group also suggested comparable degrees of clinical severity. None of the patients had conjugate gaze palsy. Characteristics of the brain damaged groups, including total number of target omissions summed for the four cancellation tasks described below, are summarised in table 1. Informed consent was obtained from all subjects prior to testing.

Materials Four conditions of a visual target cancellation task were designed by varying two dimensions: stimulus material (letters vs geometric shapes) and array (random vs structured). ${ }^{8}$ The stimuli were displayed on a $21.6 \times 27.9 \mathrm{~cm}$ sheet of paper in each of the four conditions. In each, there were 60 targets interspersed among over 300 foils. Thirty targets were to the left of the centre of the page and 30 to the right in symmetrical locations with respect to the horizontal and vertical axes of the page. The targets were in the same locations in all four conditions. In the structured array, stimuli were arranged linearly in columns and rows; in the random array, the non-target stimuli were positioned irregularly around the target positions (figs 1,2 ).

Procedures In each condition, subjects were instructed to mark the appropriate targets (the letter " $A$ " or an open circle with radiations and a single slanted line). Coloured pencils were supplied by the examiner, a different colour after every
10-15 targets marked, so that the origin and degree of organisation of the search could be assessed. Control subjects were allowed 2 minutes to complete each form, but most of them finished before this time. A time limit was not imposed on the patients since several in the left lesion group either had weakness in the preferred hand or had to use the nonpreferred hand. We did not control for the hand used but considered that the right lesion group would have been given the advantage since they all used the preferred hand. Furthermore, four subjects not included in this study because they were left-handed (two patients with left sided lesions and two control subjects) performed no differently from their respective right-handed counterparts.

The four forms were presented in a fixed order: the two random forms preceded the two structured forms and shapes preceded letters. While this may have introduced a bias, it was considered important not to influence the subjects' spontaneous search strategy by beginning with structure and familiar symbols.

For every subject, the data for each condition consisted of the total number of target omissions calculated separately for the left and right sides of the page and ratings of the search strategy. Two features of the search strategy were noted. The origin of the search, marked by the first target circled, was designated either "right" or "left" with respect to the centre of the page. The degree of organisation was rated "systematic" if the search proceeded in successive vertical or horizontal sweeps of the page (including an alternating "oxplough" pattern) and "unsystematic" if the subject proceeded in any other manner. The time required to complete each of the four conditions was available for some of the subjects 
total number of targets omitted, summed across all test forms, was 93.5 (65.5 on the left side of the page, 28.0 on the right) for the right lesion group and 12.2 ( 2.6 left, 9.6 on the right) for the left lesion group (table 2). Control subjects omitted an average total of $5 \cdot 5$ targets (3.0 left, 2.5 right).

Contralateral inattention was observed in each group of patients as reflected in a significant Group X Hemispace interaction $[F(1,14)=34 \cdot 77, p<0.0001]$. Post hoc comparisons revealed that the right lesion group omitted more targets in the left hemispace (an average of 16.4 per test form) than in the right (7.0 per test form, $p<0.001$ ) while patients with left hemispheric lesions omitted more targets in the right hemispace $(2.4$ per test form) than in the left $(0.6$ per test form, $p<0.05)$.

Each group of patients omitted more targets on the two shape tasks (mean total omissions right lesions $53 \cdot 1$; left lesions 6.8) than on the two letter tasks [right lesions 40.4; left lesions 5.4: Material main effect: $F(1$, $14)=9.31, p=0.009]$. In each group of patients, more targets were omitted in the two random arrays (right lesions 48.8; left lesions 7.0) than in the two structured arrays (right lesions $44 \cdot 7$; left lesions $5 \cdot 2$ ) [Array main effect: $\mathrm{F}(1,14)=9 \cdot 66, \mathrm{p}=0.008$ ].

(2) Left lesion group vs normal control group There was no significant difference between the left lesion

(seven in the left lesion group, four in the right, six controls) and for an additional five control subjects from another study.

\section{Results}

\section{A. Severity of neglect measured by the number of target omissions}

The following analyses were performed on the number of target omissions: (1) patients with right and left sided lesions were compared; (2) patients with left sided lesions were compared with control subjects; (3) performance within each of the two groups of patients was examined in two separate analyses. Because there was no overlap between the right lesion and control groups with respect to the number of targets omitted by each, they were not compared statistically. The effect of sensory deficits on performance is also described.

(1) Right lesion group vs left lesion group The number of target omissions as a function of subject group (right vs left lesion), material (shapes vs letters), array (random vs structured) and hemispace (right vs left side of the page) was examined by analysis of variance. Data were first subjected to a square root transformation but raw scores are reported in the text.

Subjects with right hemispheric lesions omitted more targets on both sides of the page in all conditions than did subjects with left hemispheric lesions [Group main effect: $F(1,14)=12.75, p<0.003$ ]. The average group and the control group with respect to the total number of target omissions. In fact, with the exception of subject 8 (table 1), the number of target omissions was exceedingly small in patients with left sided lesions. In contrast to controls, however, patients with left sided lesions tended to omit more targets on the right side of the page than on the left [Group $X$ Hemispace interaction: $F(1,15)=5.63, p=0.032$ ].

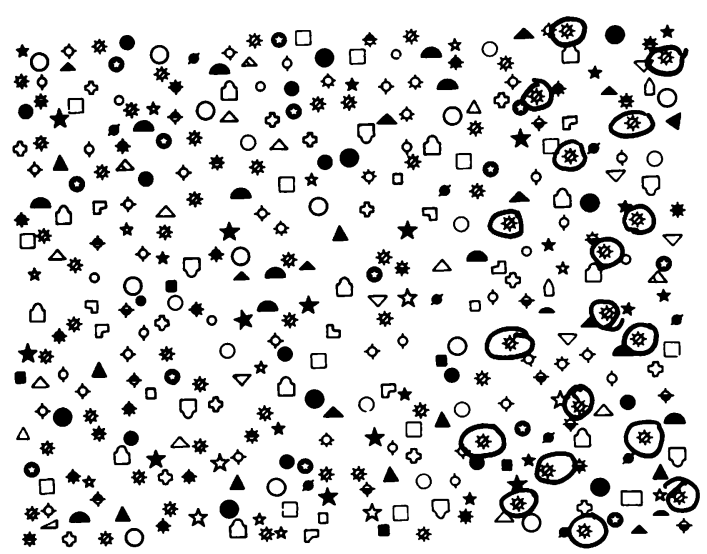

Fig 1 The random shape condition of the visual target cancellation task, taken from a patient with a large right hemispheric lesion (Subject No 15, table 1). Detected targets are circled. Neglect of the left hemispace is dramatic. 
(a)

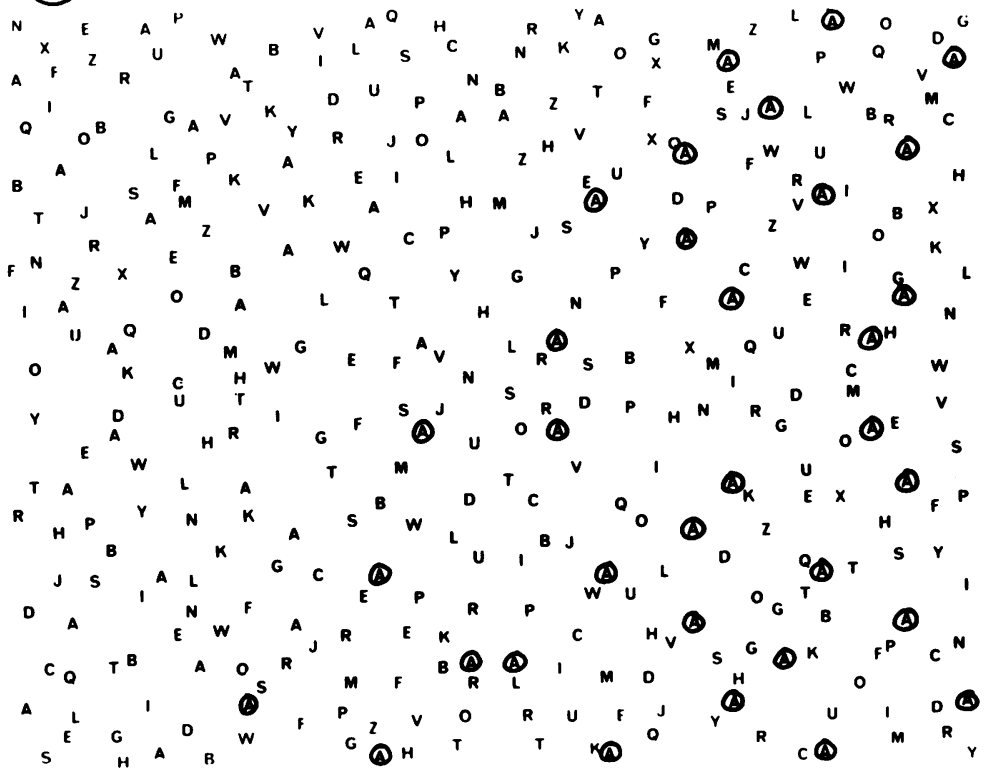

(b)

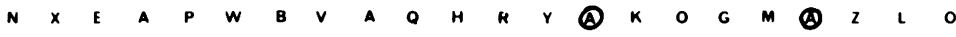

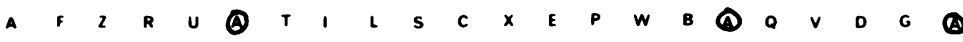

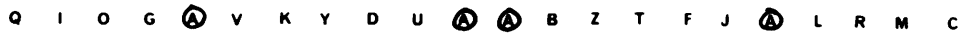

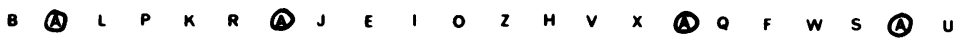

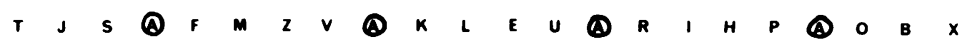

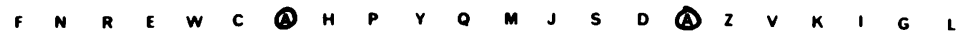

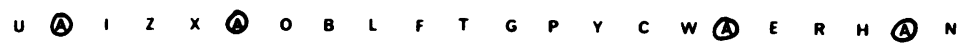

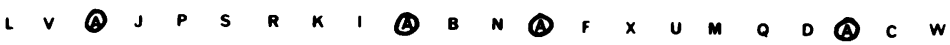

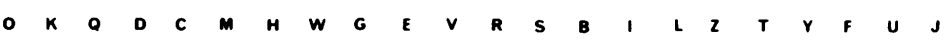

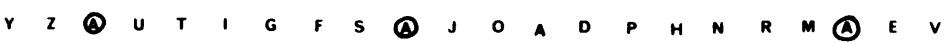

$$
\begin{aligned}
& \text { E (4) } W \quad H \quad R \quad \text { (4) } L \\
& \begin{array}{llllllllllllllllllllll}
R & T & P & Y & N & K & \mathbb{A} & S & W & L & u & C & a & E & H & A & f & B & J & 0 & z & 1
\end{array}
\end{aligned}
$$

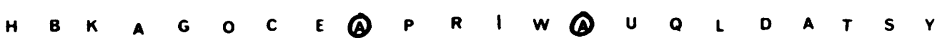

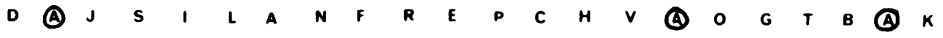

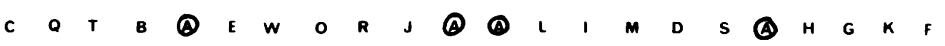

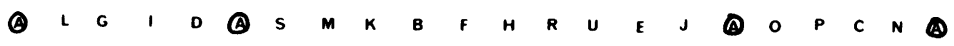

$$
\begin{aligned}
& \begin{array}{llllllllllllllllllllll}
S & E & H & (4) & B & W & F & P & \text { (A) } & G & Z & T & K & \text { (A) } & 0 & Y & R & C & \text { (4) } & U & I & M
\end{array}
\end{aligned}
$$

Fig 2 Performance on the random and structured letter cancellation tasks of a patient with a right frontal infarct (Subject No 12, table 1). Left hemispatial neglect is significantly reduced with the structured stimulus array (bottom). Target detections on the ipsilateral (right) side of the page was also impaired. 
Table 2 Mean total number of target omissions per hemispace summed across the four forms of the Cancellation Test

\begin{tabular}{llll}
\hline & $\begin{array}{l}\text { Right lesion } \\
(N=8)\end{array}$ & $\begin{array}{l}\text { Left lesion* } \\
(N=8)\end{array}$ & $\begin{array}{l}\text { Control } \\
(N=9)\end{array}$ \\
\hline $\begin{array}{l}\text { Left hemispace } \\
\text { Right hemispace }\end{array}$ & 65.5 & 2.6 & 3.0 \\
\hline
\end{tabular}

*The average number in this group was inflated by the performance of one patient (No 8, table 1).

Although the left lesion group omitted more targets in the random arrays than in the structured arrays, the interaction between Subject group and Array did not reach significance $(p=0.06)$. In all other respects, the performance of the left lesion group was similar to that of the control subjects whose performance was not influenced by either material or array.

(3) Within group comparisons of the effect of test form In patients with right sided lesions, the total number of target omissions (left and right sides of the page combined) differed significantly on the four forms of the cancellation test $(F(3,7)=8.42, p<$ 0.001 ). More shapes (mean total omissions, 28.0 ) than letters (mean total, 16.8) were omitted when the two structured forms were compared (Newman-Keuls, $p$ $<0.025$ ). In addition, more targets were omitted in the random array (mean total, 23.6) than in the structured array (mean total, 16.8, p < 0.025) when the two letter tasks were compared. These results show that the contribution of material (shapes vs letters) is most conspicuous when the less difficult structured forms are compared, whereas the contribution of array (random vs stifuctured) is most obvious when the less difficult letter tasks are compared (tables 3, 4). Fig 2 illustrates the performance of a patient with a right frontal lesion on the two forms of the letter cancellation task. Neglect is dramatic in the random array but diminishes in the structured array.

A similar comparison of the four forms of the cancellation task within the left lesion group yielded no significant differences. The mean total omissions were 3.6 for random shapes, 3.4 for random letters, 3.2 for structured shapes and 2.0 for structured letters. The absence of array and material effects should be interpreted with caution in view of the small number of total omissions in this group of patients but it reiterates the findings from the comparison reported above between the left lesion and control groups.

(4) Contribution of visual field defects and visual extinction to target omissions The small number of subjects prevented a conclusive analysis of the effect of visual field defects on performance. However, neglect was evident even in patients without hemianopia. Patients with right sided lesions and hemianopia $(\mathbf{N}=$ 2) omitted a total average of 111.5 targets (summed across all four conditions) compared with an average of 87.5 in patients without hemianopia $(N=6)$. In patients with left sided lesions and hemianopia $(\mathbf{N}=$ 3 ), a mean total of 24.3 targets were omitted, far fewer than the number omitted by patients with right sided lesions and no hemianopia. In those with left sided lesions and no hemianopia $(\mathrm{N}=5)$, the mean total was $5 \cdot 0$. Thus, a visual field defect may contribute to a general increase in the number of target omissions but is not a necessary condition for the emergence of neglect. Similarly, target omissions were observed in patients with full visual fields and no evidence of sensory extinction during bilateral simultaneous stimulation (patients 10,13, table 1). Thus hemisensory extinction and target omissions are not necessarily interdependent.

B Time per detected target as a function of stimulus material and array

The time to complete each of the four conditions had been recorded for some of the subjects (seven left sided lesions, four right sided lesions, six controls) and was available for an additional five control subjects from another study. In each condition, the total time taken for the task was divided by the number of targets detected in order to determine the time per detected target.

(1) Within group comparison of the effect of test form Within each of the three subject groups there were no differences in the time required per detected target simply as a function of stimulus material or array. Thus, the findings reported above on the number of target omissions for the patients with right sided lesions could not be attributed to differences in the speed of target detection on each form.

(2) Between group comparison of time per detected target A comparison among the three groups revealed differences with respect to the time per detected target on both the shape $[F(2,19)=14.90$, $p=0.001]$ and letter $[F(2,19)=7.53, p<0.004]$ cancellation tasks. Patients with right sided lesions required more time to detect shapes than did patients with left sided lesions (an average of $6.63 \mathrm{~s}$ per target

Table 3 Comparison of target omissions on the structured shape and structured letter tasks

\begin{tabular}{llll}
\hline & $\begin{array}{l}\text { Right lesion } \\
(N=8)\end{array}$ & $\begin{array}{l}\text { Left lesion } \\
(N=8)\end{array}$ & $\begin{array}{l}\text { Control } \\
(N=9)\end{array}$ \\
\hline $\begin{array}{c}\text { Shapes } \\
(\text { Total }=60)\end{array}$ & 28.0 & 3.2 \\
$\begin{array}{c}\text { Letters } \\
(\text { Total }=60)\end{array}$ & 16.8 & 2.0 & 1.9 \\
\hline
\end{tabular}

*NS = Not significant. 
Table 4 Comparison of target omissions on the random and structured letter tasks

\begin{tabular}{llll}
\hline & $\begin{array}{l}\text { Right lesion } \\
(N=8)\end{array}$ & $\begin{array}{l}\text { Left lesion } \\
(N=8)\end{array}$ & $\begin{array}{l}\text { Control } \\
(N=9)\end{array}$ \\
\hline $\begin{array}{l}\text { Random } \\
(\text { Total = 60) }\end{array}$ & 23.6 & 1.7 \\
$\begin{array}{c}\text { Structured } \\
\text { (Total = 60) }\end{array}$ & 16.8 & 2.0 \\
\hline
\end{tabular}

*NS = Not significant.

compared with $4.03 \mathrm{~s}, \mathrm{p}<0.025$ ). However, the right and left lesion groups did not differ significantly from one another in the speed of letter detection ( $5.6 \mathrm{~s}$ per letter in each group). The control subjects were significantly faster than either group of patients $(p<$ 0.01 ) on both the shape (average time, $1.45 \mathrm{~s}$ per detected target) and letter (1.39 s) tasks. The time per detected target was generally faster in the structured arrays than in the random arrays for both groups of patients but the difference was not statistically significant.

\section{Search strategies}

(1) Origin of search and degree of organisation With few exceptions, control subjects began their search on all forms of the cancellation task in the upper left hand corner and proceeded in systematic horizontal of vertical sweeps of the page. The search strategy of the left lesion group did not differ significantly from that of the normal control group with respect to either origin or degree of organisation. However, the search strategy of patients with right sided lesions was quite different. Table 5 compares the frequency of types of search strategies in the two groups of patients on each form of the cancellation task.

With respect to the origin of the search (left vs right side of the page) patients with right hemispheric lesions showed a strong tendency to initiate the search on the right side of the page. The extent to which the search was systematic differed significantly between the two groups of patients when the array was random. Under that condition, patients with right sided lesions tended to conduct an unsystematic search while those with left sided lesions employed a systematic search. The search strategy of patients with right hemispheric lesions became more systematic on the structured forms.

(2) Target omissions and time per detected target as a function of search strategy The average number of target omissions was calculated for the tasks in which an unsystematic approach was adopted and also for those in which a systematic strategy was used, ignoring material and array. The eight patients with right sided lesions collectively produced 32 forms. On 19 of these an unsystematic approach was used; on the remaining 13 , the approach was systematic. The number of target omissions was significantly greater when the approach was unsystematic (mean omissions, 31.9) than when it was systematic (mean omissions, 10.9; $t(30)=3.92$, $\mathrm{p}<0.001$ ). Furthermore, in those subjects for whom time had been recorded, target detection was faster during systematic. search (average time per detected target $=3.3 \mathrm{~s}$ ) than during unsystematic search (average time, $7.5 \mathrm{~s}, \mathrm{t}(13)=9.5, \mathrm{p}<0.0001$ ).

To summarise: (1) patients with right hemispheric lesions omitted more targets on each side of the page (but especially in the contralateral left side) than did patients with left hemispheric lesions, regardless of stimulus material or array; (2) patients with left hemispheric lesions omitted more targets than did normal control subjects only on the right side of the page; (3) geometric shapes and a random stimulus array generally prompted more target omissions in each group of patients than did letters and a structured array; however, when each group was analysed individually, stimulus properties affected target detection only in patients with right sided lesions; (4) lesions in the right hemisphere (but not in the left) caused a rightward shift in the origin of the search; (5) normal control subjects as well as those with left hemispheric lesions could impose a systematic search even when the stimulus array was random, whereas patients with right hemispheric lesions were less able to do so; (6) a systematic search strategy in patients with right sided lesions was associated with fewer target omissions and faster target detection.

\section{Discussion}

Four visual target cancellation tasks in which stimulus material and array were varied revealed distinct differences between patients with right sided and left

Table 5 Frequency of search strategies employed by braindamaged subjects on the four forms of the cancellation task

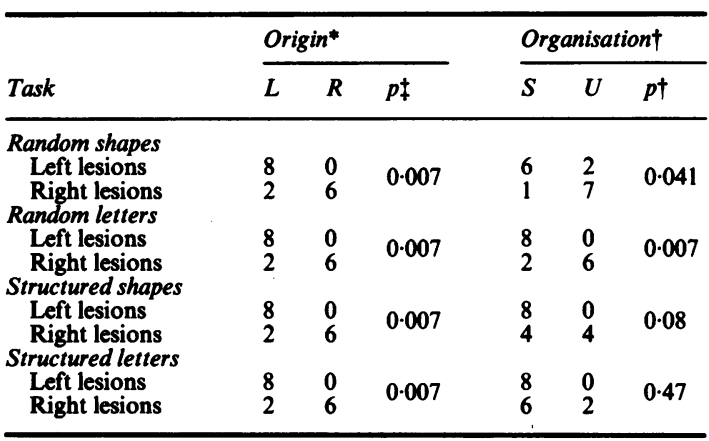

${ }^{*} L=$ left side of the page; $R=$ right side of the page. †S = systematic; $U=$ unsystematic. $\ddagger$ Based on Fisher exact test. 
sided cerebral lesions. In line with previous observations $^{9-20}$ marked contralateral hemispatial inattention, as measured by the number of target omissions, was noted only in patients with right cerebral lesions. Contralateral inattention was also evident in patients with left cerebral lesions but was much less severe. Furthermore, patients with right sided lesions also omitted targets in the hemispace ipsilateral to the lesion. This finding has been noted by others $^{61421}$ and its implications for a theory of right cerebral dominance in the spatial distribution of attention have been discussed in detail elsewhere. ${ }^{21}$

The number of target omissions differed significantly on the four forms of the cancellation test only in patients with right sided lesions. For this group, stimulus material and array interacted in a complex manner to influence target detection. When letters were the stimuli, target detection was more effective in the structured array than in the random array. Letter detection was better than shape detection but only when the influence introduced by the array was eliminated by comparing the two structured forms. Patients with left sided lesions, in contrast, did not display significant differences among the four forms with respect to target omissions. The absence of such differences in this group may reflect a "floor" effect arising from the generally small number of target omissions.

Within each of the three subject groups, the average time taken to detect a single target stimulus did not change as a function of stimulus material or array. Thus, the effect of stimulus material and array on target detection observed in patients with right sided lesions could not be ascribed to differences in the speed of single target detection. Comparisons among the groups revealed that patients with right sided lesions required more time per detected target than patients with left sided lesions when the stimuli were shapes and did not differ from patients with left sided lesions when the stimuli were letters. Control subjects were faster than either group of patients with both types of stimuli.

Initially it may appear as if our findings are in conflict with those of others. ${ }^{36}$ Heilman and Watson ${ }^{3}$ observed that patients with right cerebral lesions omitted fewer targets on a nonverbal than on a verbal cancellation task. However, the stimuli used in that study may have been easier to detect than those of the present study, making it difficult to compare the findings. Caplan, ${ }^{6}$ in contrast, reported that the severity of neglect did not vary as a function of stimulus material in patients with either right or left sided lesions. The nature of the stimuli used in that study may have accounted for the divergence from our findings since the nonverbal stimuli included Greek letters and nameable symbols (such as arrows). Our findings are more in agreement with Leicester et al ${ }^{\prime}$ who reported an increase in the severity of neglect for nonverbal material in patients with right sided lesions. However, a definitive elucidation of the effect of material will need to be based on experiments in which the verbal and non verbal stimuli are designed to be of equal difficulty.

The exploratory strategy differentiated between patients with right and left sided lesions. Patients with left sided lesions, like the control subjects, initiated the search on the left side of the page and spontaneously adopted a systematic search pattern even with the unstructured forms. In contrast, patients with right cerebral lesions typically began on the right side of the page and searched erratically, particularly when the stimulus array was random. Most of them, however, adopted a systematic approach with the structured arrays. They detected more targets and the time per detected target was shorter when the search strategy was systematic than when it was disorganised. It is quite likely, therefore, that erratic search in patients with right sided lesions, contributes to the severity of hemispatial inattention.

The fixed order in which the four forms were presented (random shapes, random letters, structured shapes, structured letters) could have introduced a practice effect which might have contributed to the current findings regarding the effects of material and array on the number of target omissions. Presenting the forms in random sequence would have been preferable but would not have permitted an assessment of the subjects' spontaneous approach in the absence of prior exposure to stimulus structure. While practice could have influenced the number of target omissions on the four forms, it is less likely that this was a factor influencing the patients' search strategies.

Chedru et $a l^{7}$ reported that eye movements, oculographically recorded during visual exploration tasks, were unsystematic in patients with right lesions and systematic in those with left lesions. Chain et al, ${ }^{17}$ used eye movements to measure the severity of neglect in patients with unilateral lesions in response to a variety of visual stimuli. However, only the time spent in each visual hemifield was recorded and there was no indication whether the patterns of scanning differed with different visual stimuli. One interesting finding in that study was that patients with right hemispheric lesions manifested neglect on tasks requiring scanning of a random array or examination of geometric shapes, portraits and scenes, but showed no neglect when asked to read a paragraph of text. It was suggested that neglect was reduced in this condition because of the intact left hemisphere's superiority for processing verbal information. However, in the light of our findings, another explanation for reduced neglect on the reading task is that the structured text 
induced more systematic and efficient scanning.

The contribution of defective visual search to neglect behaviour associated with right hemispheric lesions has been recently questioned. Gainotti et al ${ }^{22}$ reported that a task requiring visual exploration elicited neglect in patients with lesions in either hemisphere while a task that minimised eye movements and emphasised foveation resulted in neglect only in patients with right sided lesions. On the basis of these findings, it was proposed that the neglect in patients with right hemispheric damage reflects the inability to extract information from the left hemispace and is not due to defective visual search. We agree that there is a perceptual component in left hemispatial neglect but our results also show that defective search strategies are likely to provide an additional factor influencing its severity.

The spatial distribution of attention is a complex function composed of perceptual-representational, exploratory-motor, motivational and arousal components and is mediated by an equally complex and widespread neural network. ${ }^{2324}$ Different measures of neglect each emphasise a different component of this network. Our results, based on tasks that emphasise visual exploration, suggest that damage to the right hemisphere (but not to the left) impairs search strategies and that this may contribute to the severity of the visual inattention observed in patients with such lesions. Our observations also suggest that a tendency to initiate search on the right or to scan a visual array in an unsystematic manner may serve, in the absence of other more obvious clinical findings, as a diagnostic sign of right hemispheric damage.

We thank Joan Guinessey, David Benson and Laura Kramer, MD for assistance with data collection, Rick Plourde for photography and Della Grigsby and Leah Christie for secretarial assistance, Dr Michael Feldstein and Harriet Peterson of the Biostatistics Department of the Harvard School of Public Health for statistical consultation. Dr Bernard Ransil also provided consultation and some of the statistical analyses were carried out on the Core Lab computer facilities at the Beth Israel Hospital. This work was supported in part by Alzheimer's Disease Research Center grant 1P50 AGO5134-01 from the National Institute on Aging to the Harvard Medical School, a grant from the McKnight Foundation and the Jacob Javitz Neuroscience Investigator award to $\mathrm{Dr}$ Mesulam, and Grant RR-01032 from the General Clinical Research Centers Program of the Division of Research Resources, NIH.

\section{References}

1 Leicester J, Sidman M, Stoddard LT, Mohr JP. Some determinants of visual neglect. J Neurol Neurosurg Psychiatry 1969;32:580-7.

2 Colombo A, De Renzi E, Faglioni P. The occurrence of visual neglect in patients with unilateral cerebral disease. Cortex 1976;12:221-31.

3 Heilman KM, Watson RT. Changes in the symptoms of neglect induced by changing task strategy. Arch Neurol 1978;35:47-49.

4 Kinsbourne $M$. The cerebral basis of lateral asymmetries in attention. Acta Psychol 1970;33:193-210.

5 Kinsbourne $M$. A model for the mechanism of unilateral neglect of space. Trans Am Neurol Assoc 1970;95:143-6.

6 Caplan B. Stimulus effects in unilateral neglect. Cortex 1985;21:69-80.

7 Chedru F, Leblanc M, Lhermitte F. Visual searching in normal and brain-damaged subjects (Contribution to the study of unilateral inattention). Cortex 1973;9:94-111.

8 Weintraub S, Mesulam M-M. Mental state assessment of young and elderly adults in behavioral neurology. In: Mesulam M-M, ed. Principles of Behavioral Neurology. Philadelphia: FA Davis, 1985:71-123.

9 Brain WR. Visual disorientation with special reference to lesions of the right cerebral hemisphere. Brain 1941;64:224-72.

10 Critchley M. The Parietal Lobes. London: Edward Arnold, 1953.

11 Denny-Brown D, Chambers RA. The parietal lobe and behavior. Annals of Research in Nervous and Mental Disease 1958;36: 35-117.

12 De Renzi E, Faglioni P, Scotti G. Hemispheric contribution to exploration of space through the visual and tactile modality. Cortex 1970;6:191-203.

13 Gainotti G, Messerli P, Tissot R. Qualitative analysis of unilateral spatial neglect in relation to laterality of cerebral lesions. $J$ Neurol Neurosurg Psychiatry 1972;35:545-50.

14 Albert ML. A simple test of visual neglect. Neurology 1973;23: $658-64$.

15 Oxbury JM, Campbell DC, Oxbury SM. Unilateral spatial neglect and impairments of spatial analysis and visual perception. Brain 1974;97:551-64.

16 Zarit SH, Kahn RL. Impairment and adaptation in chronic illness: Spatial inattention. $J$ Nerv Ment Dis 1974;159:63-72.

17 Chain F, Leblanc M, Chedru F, Lhermitte F. Negligence visuelle dans les lesions posterieures de l'hemisphere gauche. Rev Neurol (Paris) 1979;135:105-26.

18 Heilman KM, Valenstein E. Mechanisms underlying hemispatial neglect. Ann Neurol 1979;5:166-70.

19 Damasio AR, Damasio H, Chang Chui H. Neglect following damage to frontal lobe and basal ganglia. Neuropsychologia 1980;18:123-32.

20 Denes G, Semenza C, Stoppa E, Lis A. Unilateral spatial neglect and recovery from hemiplegia. Brain 1982;105:543-52.

21 Weintraub S, Mesulam M-M. Right cerebral dominance in spatial attention: Further evidence based on ipsilateral neglect. Arch Neurol 1987;44:621-5.

22 Gainotti G, D'Erme P, Monteleone D, Silveri MC. Mechanisms of unilateral spatial neglect in relation to laterality of cerebral lesions. Brain 1986;109:599-612.

23 Mesulam M-M. A cortical network for directed attention and unilateral neglect. Ann Neurol 1981;10:309-25.

24 Mesulam M-M. Attention, confusional states, and neglect. In: Mesulam M-M, ed. Principles of Behavioral Neurology. Philadelphia: F A Davis, 1985:125-68. 